\title{
Colloid Cyst Presenting as Head Injury
}

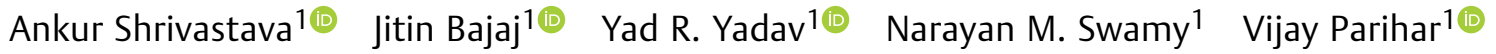 \\ Shailendra Ratre ${ }^{1}$ Mallika Sinha ${ }^{1}$ Ketan Hedaoo ${ }^{1}$ Ambuj Kumar ${ }^{1}$ Pankaj Singh Chauhan $^{1}$ P $^{\circ}$ \\ ${ }^{1}$ Department of Neurosurgery, Super Speciality Hospital, Netaji Subhash \\ Chandra Bose Medical College, Jabalpur, Madhya Pradesh, India \\ Address for correspondence Yad R. Yadav, MCh, Department of \\ Neurosurgery, Super Speciality Hospital, Netaji Subhash Chandra \\ Bose Medical College, Jabalpur 482003, Madhya Pradesh, India \\ Indian J Neurosurg 2023;12:76-78. \\ (e-mail: yadavyrns@gmail.com).
}

\begin{abstract}
Colloid cysts are mucous- or hyaloid-filled lesions with an outer fibrous layer. These are rare developmental and nonneoplastic malformation. They may be found incidentally, while some cases may present with intermittent headache, rapid neurologic deterioration, drop attacks, and even sudden death. Early recognition of this disease may result in lesser mortality. Here, we present a 22-year-old male with a history of fall while driving his two-wheeler. A diagnosis of colloid cyst of the third ventricle with a head injury was made. Colloid cyst presenting with a head injury is exceedingly rare with only five case reports in the literature. This report may help to support surgical intervention in an asymptomatic patient, as the cyst can predispose head injury with serious consequences. It also stresses the importance of a high degree of suspicion when there is any well-defined radiological abnormality in the region of the third ventricle. A colloid cyst may easily be confused with intracranial hemorrhage due

\section{Keywords}

- colloid cyst

- craniocerebral trauma

- endoscopy to hyperdensity in head trauma. It can be distinguished by the presence of an accompanying traumatic lesion. The cyst usually has well-defined round margins, and the morphologic appearance and density do not change on a follow-up computed tomography (CT) scan, whereas in hemorrhage the density usually regresses with clinical improvement on serial imaging.
\end{abstract}

\section{Introduction}

Colloid cysts account for nearly 0.2 to $2 \%$ of all intracranial neoplasms and around 15 to $20 \%$ of intraventricular masses. ${ }^{1}$ The presentation may vary from asymptomatic to features of intermittent intracranial hypertension or chronic hydrocephalus to rapid neurologic deterioration, drop attacks, and sudden death. Colloid cyst presenting as a head injury is extremely rare with only four case studies involving five patients reported so far. ${ }^{1-4}$

\section{Case Report}

A 22-year-old male presented in the emergency with a history of fall while driving a two-wheeler. The pillion rider noticed

article published online July 10,2022
DOI https://doi.org/ 10.1055/s-0041-1729516. ISSN 2277-954X. that the patient's body stiffened before fall. There was no history of headache, syncope, or congenital heart disease. He sustained a severe head injury and a right tibia fracture. His Glasgow coma score was E1VtM3. Both pupils were sluggishly reacting to light. An electrocardiogram and biochemistry profile were normal. A noncontrast computed tomography (NCCT) of the brain revealed a well-defined circular mass of $12 \mathrm{~mm}$ in diameter located in the third ventricle (- Fig. 1A). Both lateral ventricles were dilated with hemorrhage in occipital horns (-Fig. 1B). A nondisplaced left frontal fracture with underlying moderate-sized acute extradural hematoma (EDH) with a 2-mm midline shift was also seen. Basal cisterns were obliterated. The patient underwent left frontal craniotomy with evacuation of EDH and endoscopic

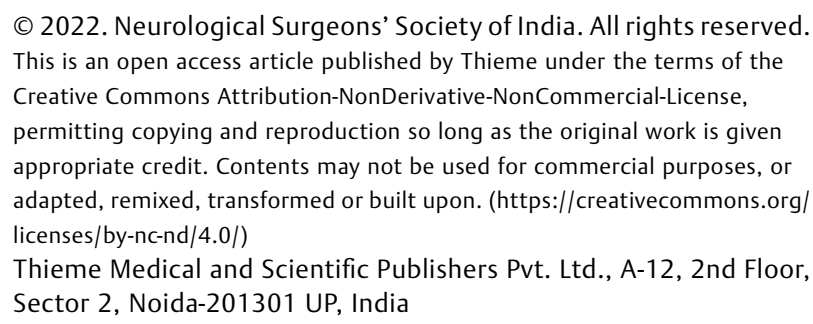




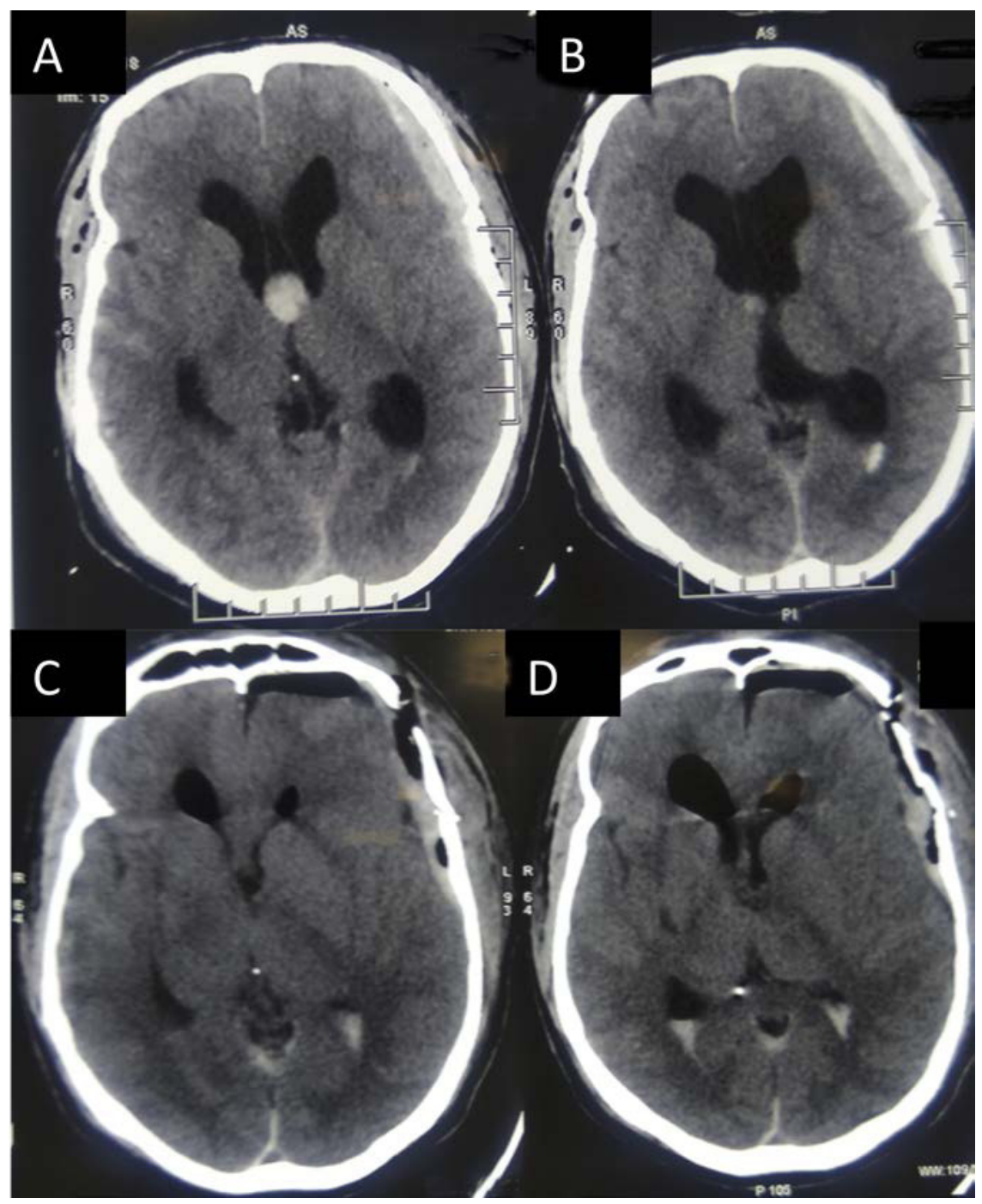

Fig. 1 (A) Preoperative NCCT brain shows left frontal EDH, cerebral edema, and a colloid cyst in the anterior third ventricle. (B) Preoperative NCCT brain showing intraventricular bleed, associated hydrocephalus, and cerebral edema. (C) Postoperative scan showing complete excision of the cyst. (D) Postoperative image showing minimal intraventricular air. EDH, extradural hematoma; NCCT, noncontrast computed tomography.

excision of the colloid cyst using a tubular retractor (-Fig. 2). The postoperative scan shows the complete excision of the cyst (-Fig. 1C) with minimal pneumoventricle (-Fig. 1D). Antiedema measures were taken. The patient was managed
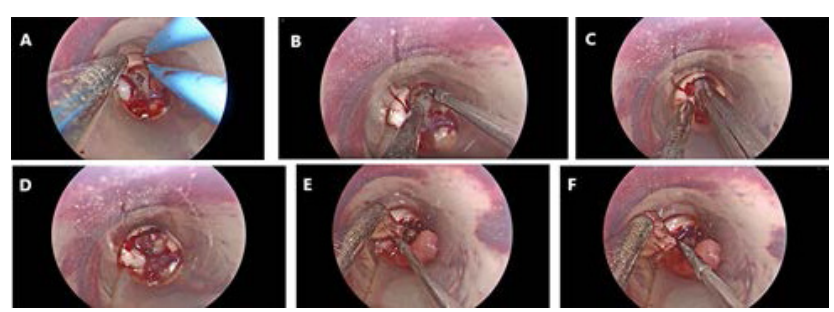

Fig. 2 Intraoperative images showing the use of tubular retractor during endoscopic excision of the colloid cyst with bimanual dissection. (A) Colloid cyst visible at the foramen of Monro (FoM), and cyst wall being cauterized with bipolar cautery to shrink it. (B) Decompression was done by aspirating the cyst contents. (C) Bimanual technique used to dissect the cyst. (D) Decompressed cyst. (E) Sectioning the cyst from its roof. (F) Cyst being removed. in the intensive care unit postoperatively, but he expired on the fourth postoperative day.

\section{Discussion}

Colloid cysts may present with headache, gait disturbance, and short-term memory loss. Some patients may present with paroxysmal symptoms due to intermittent blockage at the foramen of Monro. ${ }^{5,6}$ Sudden ventricular obstruction and resultant rapid rise in intracranial pressure can lead to herniation and sudden death. ${ }^{5}$

Colloid cyst presenting as a head injury is reported rarely. ${ }^{1-4}$ Two cases by Cultrera et al presented with delayed neurological deterioration following minor head trauma. ${ }^{3}$ NCCT head revealed a third ventricular colloid cyst and hydrocephalus. Both patients underwent bilateral ventriculoperitoneal shunts and improved thereafter. Barker et al presented a young patient with sudden onset global alexia and agraphia after minor head trauma. ${ }^{2}$ Imaging 
showed a third ventricular colloid cyst with splayed fornix. Morris and Santoreneos reported an 11-year-old boy who suffered a minor head injury following a fall while playing. ${ }^{4}$ NCCT scan done for memory loss revealed a homogeneous cystic lesion within the velum interpositum in the roof of the third ventricle. The patient underwent resection of the lesion resulting in memory improvement. In the report by Buyukkaya et al, the lesion was initially considered as hemorrhage. ${ }^{1}$ Follow-up with serial NCCT scanning made a diagnosis of a colloid cyst as the lesion did not change in density or size.

Our report is different from previous cases. All previously reported patients had mild head injury without any mortality, whereas our patient died due to serious head injury. Although the cyst was detected after a head injury, the cause of the fall was possibly the cyst. There was no circumstantial evidence on the road that could have caused a fall except the drop attack due to cyst. This report signifies that the cyst can result in serious head injuries. In head trauma, a colloid cyst may easily be confused with intracranial hemorrhage as both appear hyperdense on NCCT scan. These can be distinguished by smooth rounded appearance of cyst, and gradual change of density in serial scans in hemorrhage. A high degree of suspicion should always be there.

Treatment options include observation, stereotactic aspiration, microscopic or endoscopic excision, and shunt. For incidentally detected small cysts of less than $1 \mathrm{~cm}$ without hydrocephalus, a conservative approach is reasonable. ${ }^{5,7}$ Surgical resection or shunt were performed in symptomatic patients of more than $1 \mathrm{~cm}$ in size, or those associated with hydrocephalus. ${ }^{3,4}$ Microsurgical approaches (transcortical and transcallosal) are commonly used, but recently, the endoscopic transcortical approach with or without tubular retractors has become increasingly common. ${ }^{8}$ These techniques are found to have lower rates of infection, seizures, postoperative infarct, and shunt dependence rates. Microsurgical advocates continue to point to a higher gross total resection and lower recurrence rates $^{9}$; however, others point no difference between the two approaches. $^{9}$

\section{Conclusion}

Colloid cysts are uncommon nonneoplastic lesions mostly found in the anterior third ventricle. These are rarely symptomatic but are known to present with sudden neurological deterioration, drop attacks, or even sudden death, and hence can put the patients at risk of accidents. It also signifies the importance of keeping a high index of suspicion, stringent follow-up, educating the patient of the possible risks, and early intervention.

Conflict of Interest

None declared.

\section{References}

1 Buyukkaya A, Buyukkaya R, Özel MA, Sarıtas A. Colloid cyst mimicking intracranial hemorrhage after head trauma. Am J Emerg Med 2015;33(02):314.e1-314.e2

2 Barker LA, Morton N, Romanowski CAJ, Gosden K. Complete abolition of reading and writing ability with a third ventricle colloid cyst: implications for surgical intervention and proposed neural substrates of visual recognition and visual imaging ability. BMJ Case Rep 2013:2013

3 Cultrera F, Parisi G, Platania N, Consoli V, Albanese V. Neurological deterioration after head trauma in patients with colloid cysts of the 3rd ventricle. Two case histories. J Neurosurg Sci 2004;48(02): 67-70

4 Morris TC, Santoreneos S. Colloid cyst of velum interpositum: a rare finding. J Neurosurg Pediatr 2012;9(02):206-208

5 Yadav YR, Yadav N, Parihar V, Kher Y, Ratre S. Management of colloid cyst of third ventricle. Turk Neurosurg 2015;25(03): 362-371

6 Lagman C, Rai K, Chung LK, et al. Fatal colloid cysts: a systematic review. World Neurosurg 2017;107:409-415

7 de Witt Hamer PC, Verstegen MJT, De Haan RJ, et al. High risk of acute deterioration in patients harboring symptomatic colloid cysts of the third ventricle. J Neurosurg 2002;96(06):1041-1045

8 Yadav YR, Parihar V, Pande S, Namdev H. Endoscopic management of colloid cysts. J Neurol Surg A Cent Eur Neurosurg 2014;75(05): 376-380

9 Sethi A, Cavalcante D, Ormond DR. Endoscopic versus microscopic transcallosal excision of colloid cysts: a systematic review in the era of complete endoscopic excision. World Neurosurg 2019;132: e53-e58 